\title{
Implementing a multidisciplinary care bundle to reduce colon surgical site infections
}

\author{
Chi-Min Park ${ }^{1,2, *}$, Tae Sun Ha ${ }^{3, *}$, Woo Yong Lee ${ }^{2}$, Doo Ryeon Chung ${ }^{4}$, Yoon Ah Park², Jong Rim Choi ${ }^{5}$, \\ Na Yeon Jeong ${ }^{5}$ \\ ${ }^{1}$ Department of Critical Care Medicine, Samsung Medical Center, Sungkyunkwan University School of Medicine, Seoul, Korea \\ ${ }^{2}$ Department of Surgery, Samsung Medical Center, Sungkyunkwan University School of Medicine, Seoul, Korea \\ ${ }^{3}$ Department of Surgery, Soonchunhyang University Bucheon Hospital, Bucheon, Korea \\ ${ }^{4}$ Department of Infectious Diseases, Samsung Medical Center, Sungkyunkwan University School of Medicine, Seoul, Korea \\ 5 Infection Control Office, Samsung Medical Center, Seoul, Korea
}

\begin{abstract}
Purpose: The aim of this study was to investigate how rates of surgical site infections (SSI) were changed over 2 years after applying colon SSI bundle in patients who underwent colon surgery.

Methods: The multidisciplinary working group developed a care bundle consisting of 8 components, including several recommendations of Surgical Care Improvement Project and monitoring of medical/surgical hand washing. We implemented the care bundle for each patient who underwent colon surgery from April 2013 to December 2014.

Results: Overall bundle compliance was $87.9 \%$ before implementation, $88.2 \%$ in 2013 , and $90.5 \%$ in 2014 . In particular, compliance of the following 3 components was substantial improved during the project period; discontinuation of prophylactic antimicrobial agent within 24 hours of surgery (from $88.3 \%$ to $100 \%$ ), surgical hand washing (from $50.0 \%$ to $78.9 \%$ ), and medical hand washing (from $74.7 \%$ to $82.8 \%$ ). The rate of SSI was $8.0 \%(12 / 150)$ during 3 months before implementation, 3.3\% (16/480) from April to December in 2013, and 2.3\% (14/607) in 2014.

Conclusion: After implementation of multidisciplinary care bundle, the compliance of each component was increased and rates of SSIs were significantly decreased compared to those before the quality improvement project.

[Ann Surg Treat Res 2020;99(5):285-293]
\end{abstract}

Key Words: Bundle, Colorectal surgery, Compliance, Surgical wound infection

\section{INTRODUCTION}

Surgical site infections (SSIs) are known to increase morbidity, hospital length of stay, readmission rates, and health care cost while the patient may suffer from permanent disability or even death [1]. SSI represents a common and important complication in colorectal surgery, occurring in $15 \%-30 \%$ of patients [2]. Therefore, reduction of SSIs, a quality metric in surgical care, has become a major target of quality improvement initiatives in patients who have undergone colorectal surgery [3]. A bundle is a structured method of improving the processes of care and patient outcomes based on a set of evidence-based interventions composed with generally 3-5 elements. The Surgical Care Improvement Project (SCIP), based on an evidence-based initiative, was launched by the Centers for Medicare and Medicaid Services in 2006 with the goal to reduce morbidity and mortality rate $25 \%$ by the year 2010 [4]. Surgical care bundles, known as SSI bundle,
Received June 1, 2020, Revised July 10, 2020, Accepted July 29, 2020

Corresponding Author: Woo Yong Lee

Department of Surgery, Samsung Medical Center, Sungkyunkwan University School of Medicine, 81 Irwon-ro, Gangnam-gu, Seoul 06351, Korea

Tel: +82-2-3410-1096, Fax: +82-2-2148-7088

E-mail:wooyong123.lee@samsung.com

ORCID: https://orcid.org/0000-0001-8758-4149
${ }^{*}$ Chi-Min Park and Tae Sun Ha contributed equally to this study as co-first authors.

Copyright (c) 2020, the Korean Surgical Society

(C) Annals of Surgical Treatment and Research is an Open Access Journal. All articles are distributed under the terms of the Creative Commons Attribution NonCommercial License (http://creativecommons.org/licenses/by-nc/4.0/) which permits unrestricted non-commercial use, distribution, and reproduction in any medium, provided the original work is properly cited. 
have been directed at reducing SSIs after the success of care bundles in reducing ventilator-associated pneumonia (VAP) and catheter-related bloodstream infection (CRBSI) [5]. SSIs have the potential to be reduced by a bundle approach because SSIs are associated with many risk factors that can be targeted with considerable evidence-based interventions deriving from the national guidelines for the prevention of SSIs [6]. Several studies identified that independent risk factors for SSI in patients undergoing colorectal surgery were body mass index, presence of diabetic mellitus, American Society of Anesthesiology (ASA) physical status (PS) classification, type of operation, duration of operation, and surgical wound class $[7,8]$.

Many hospitals have instituted colorectal SSI bundles in an effort to use evidence-based approaches with a reasonable method to reduce the incidence of SSIs. Commonly implemented interventions in many hospitals were SCIP elements consisting of prophylactic antibiotics within 1 hour before incision, use of antimicrobial agents consistent with published guidelines, discontinuation of the antimicrobial agent within 24 hours after surgery, proper hair removal with clippers instead of razors, blood glucose control for cardiac postoperative patients, and maintenance of perioperative normothermia. These bundles, however, have been encountered with varying degrees of success and effectiveness [9,10]. The objective of this study was to investigate how rates of SSI were changed over a 2-year period after applying a colon SSI bundle in patients who underwent elective colon surgery.

\section{METHODS}

\section{Study design}

Our study was a retrospective study with prospectively collected bundle registry to evaluate incidence of SSIs in elective colorectal cancer cases at Samsung Medical Center (a 1,960 bed, university-affiliated, tertiary referral hospital in Seoul, a city with 10.36 million residents, Korea). At this hospital, approximately 600-700 major elective and emergency colorectal procedures are performed annually. This study was approved by the Institutional Review Board (IRB) of Samsung Medical Center to review and publish information obtained from patient records (No. 2019-11-012-001). The IRB waived the need for written informed consent from patients because of the observational nature of this study. In addition, patients' information was anonymized and de-identified prior to analysis.

This study was begun as part of quality improvement project which usually starts in March. For definite monitoring, survey of actual performance of each bundle needed team activity and many observers, which was obtained via quality improvement project. Patients who underwent laparoscopic and open colorectal operations with data submitted to SCIP from March
2013 to December 2014 were included in this study. Procedures included anterior resection, low anterior resection, and partial or total colectomy with or without proctectomy. A baseline data collection of actual performance of each bundle before adjusting care bundle project was conducted in March 2013 for 1 month. Colon SSI bundle was then introduced and implemented, and intervention cohort data were collected from April 2013 to December 2014. A collecting of data for SSI was performed between January 2013 and December 2014. Unlike compliance with colon SSI bundle, SSI surveillance was conducted during the period 3 months preintervention from January to March 2013.

\section{Study population}

We included patients aged 18 years or older who underwent colon surgery. Patients who were younger than 18 years old, who underwent colon surgery before admission in other hospitals, who received antibiotics for preexist infection before elective colon surgery, who received operation under general or spinal anesthesia within 3 days before colon surgery, whose hospital days were within 2 days after colon surgery, who received multiple sites operation simultaneously, and those who exceeded 2 days of length for intensive care unit (ICU) stay were excluded from this study.

\section{Colon surgical site infection bundle}

The use of the bundle involved a systematic approach to improve compliance with each care bundle intervention across the phase of perioperative care. We engaged a multidisciplinary working team consisting of infection control office, colorectal surgeons, anesthesiologists, and perioperative nurses in November 2012. The team met monthly to monitor and provide feedback to improve compliance during the project period. Between meetings, subgroups worked to execute action plans and implement interventions to address the identified concerns. Education for participated medical personnel, including attending staff, fellows, residents, interns, medical students, scrub nurses, and ward nurses, regarding elements of colon SSI bundle was done before its implementation.

On the basis of SCIP measures, the colon SSI bundle consisted of 8 elements that were thought to pose minimal risk and hold potential for benefit was confirmed by the multidisciplinary team in February 2013. The 8 elements of the colon SSI bundle elements were:

1. Administration of prophylactic antibiotics within 1 hour

2. Appropriate selection of prophylactic antibiotics for surgical patients

3. Prophylactic antibiotics discontinued within 24 hours after surgery end time

4. Appropriate hair removal using single-use clipper if hair removal is needed 
5. Maintenance of normothermia in perioperative period

6. Urinary catheter removal within postoperative 2 days

7. Medical hand washing

8. Surgical hand washing

Normothermia was defined as a temperature between $36.0^{\circ} \mathrm{C}$ and $38.0^{\circ} \mathrm{C}$ during perioperative period. For perioperative antibiotics prophylaxis, all patients without an allergy received a single 1-g dose of cefotetan (second-generation cephalosporin) within 1 hour of incision. Moxifloxacin was used as an alternative when an allergy was present. If hair removal was needed, single-use clipper or hair removal cream had to be used while razor blade was not allowed. Surgical field preparation was performed in standardized fashion using $2 \%$ chlorhexidine gluconate-70\% isopropyl alcohol solution. After surgery, dressing was implemented using chlorhexidine within 48 hours from the time of surgery. Surgical hand washing is named several different terms as surgical hand antisepsis. After cleaning nails using a pick under running water, operation team then performed either an antisepsis using antimicrobial solution with running water, referred to as a traditional scrub using chlorhexidine gluconate or povidone-iodine, or an alcoholic rub without water. Medical hand washing is termed hygienic hand washing which uses antiseptic preparations containing bactericidal chemical compounds that is directly poured or otherwise applied to the hand and then rubbed into the skin until hands are dry for at least 30 seconds. Medical hands washing is indicated in the following circumstances: prior to direct contact with patients; prior to invasive or nursing activities regardless of whether gloves are used or not; prior to contact with catheters, drains, and equipment parts directly in contact with patient's tissues; and prior to contact with blood, body fluids, and operation site before wound dressing. In addition, the infection control office educated accurate method about surgical and medical hand washing, particularly regarding brushing and the duration and extent of rubbing, for residents and professors at conference time of general surgery. Compliance data with surgical and medical hand washing were monitored by the infection control office in key areas such as operating rooms and general ward.

\section{Data collection and analysis}

The following characteristics were recorded; age, sex, ASA PS classification, wound classification, type of procedure, laparoscopic vs. open, temperature at the end of surgery, duration of surgery, surgeon, anesthesiologist, admission date, date of surgery, discharge date, and development of SSIs. SSIs as defined by the National Surgical Quality Improvement Project were further classified by the Centers for Disease Control and Prevention of United States (US CDC) based on their anatomic involvement relative to the surgical wound [11]. National surveillance systems for nosocomial infection have already been developed and implemented successfully in the US and European countries [12]. We adopted the definition from US CDC used to diagnose SSI.

The investigation methods were as follows: (1) the surgeon or surgical team was directed to examine the surgical site more than 3 times per week during the hospital stay for patients who underwent operations subject to surveillance; (2) the presence of SSI was determined through direct observation of the surgical site by the surgeon or surgical team, or adjunctively with indirect methods through a review of a patient's medical record, microorganism test, or radiological data; (3) the surgeonreport survey form was completed at the first outpatient followup appointment; and (4) the surveillance period was set as 30 postoperative days.

The presence of SSI was prospectively monitored until SSI developed or 30 days had passed after surgery. Data entry and surveillance were performed by the lead surveillance nurse through direct patient contact and hospital electronic medical record (EMR). Colon SSI bundle compliance was measured by the infection control office. Direct observation of clinical practice was also undertaken by the assistant to confirm the validity of documented data. Clinical practice for hair removal using single-use clipper, medical hand washing, and surgical hand washing was directly observed by the assistant, and the others were identified via EMR. In addition, SSI rates were compared before and after implementation of the colon SSI bundle and calculated every month. Quarterly reports were established. Each case of SSIs was identified and reviewed every month with regard to elements of the bundle. Every month, the total number of documented SSIs was divided by the total number of patients at risk in that period and expressed as the overall case report. Rates for superficial, deep, and organ/space SSIs were calculated in a similar fashion.

The National Nosocomial Infectious Surveillance (NNIS) risk index, proposed by the US CDC in 1991, was developed to predict the risk of acquiring SSI in surgical patients [13]. This index consists of ASA PS classification, wound, and duration of surgical procedure. The risk index score, ranging from 0 to 3 , is the number of risk factors present among the following: ASA PS classification of III, IV, V; wound classification as contaminated or dirty-infected; and the duration of surgical procedure longer than $75 \%$ percentile of the total surgical duration.

\section{Statistical analysis}

Data are presented as medians and interquartile ranges (IQR, 25th and 75th percentiles) for continuous variables and as numbers (percentages) for categorical variables. Data were compared using Mann-Whitney U-test for continuous variables and chi-square or Fisher exact test for categorical variables. All tests were 2-sided and a P-value of $<0.05$ was considered statistically significant. Data were analyzed using IBM SPSS 
Statistics ver. 20 (IBM Corp., Armonk, NY, USA) for descriptive analysis.

\section{RESULTS}

\section{Baseline characteristics}

Between March 2013 and December 2014, a total of 1,586 who underwent laparoscopic and open colorectal operations were entered into the registry. Of these, 428 patients were excluded according to the exclusion criteria, including those with combined operation $(n=128)$, colon surgery before admission $(\mathrm{n}=17)$, receiving antibiotics for preexist infection before colon surgery $(\mathrm{n}=11)$, multiple site operation $(\mathrm{n}=247)$, hospital stay within 2 days after colon surgery $(\mathrm{n}=8)$, over 2 days of length of ICU stay $(n=6)$, and medical record miss $(n=11)$. Finally, a total of 1,158 patients were eligible for this study. Of these eligible patients, preintervention for the month of March 2013, postintervention from April 2013 to December 2013 (postintervention 1-year) and postintervention from January 2014 to December 2014 (postintervention 2-year) were 71, 480, and 607 cases, respectively. For SSI, preinterventions from
January 2013 to March 2013 were 150 cases. Fig. 1 summarizes patient flow in this study. The baseline characteristics of patients of postintervention are summarized in Table 1. There were no significant differences in baseline characteristics between postintervention 1-year and 2-year except the type of operation.

\section{Colon SSI bundle compliance and outcome}

The overall compliance in the preintervention group was $87.9 \%$. Overall compliances in the postintervention 1-year (88.2\%) and 2-year (90.5\%) groups were increased. Prophylactic antibiotics within 1 hour, appropriate hair removal, and maintenance of normothermia in the perioperative period had high compliance during the entire study period because they had already been conducted at the hospital prior to the implementation of the bundle. Discontinuation of prophylactic antibiotics within 24 hours was increased from $88.3 \%$ to $96.9 \%$ in the postintervention 1-year group and $99.7 \%$ in the postintervention 2-year group (Fig. 2A). Medical hand washing was increased from $74.7 \%$ to $75.6 \%$ (1-year) and $82.8 \%$ (2-year) while surgical hand washing was increased from $50.0 \%$ to $63.6 \%$
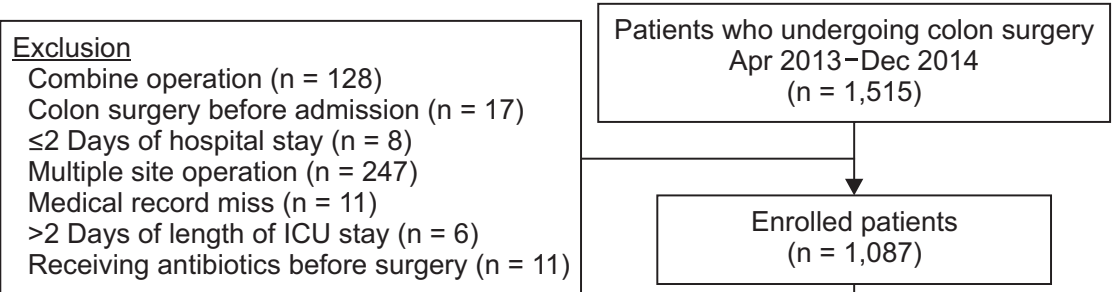

Before implementation $(n=71)$

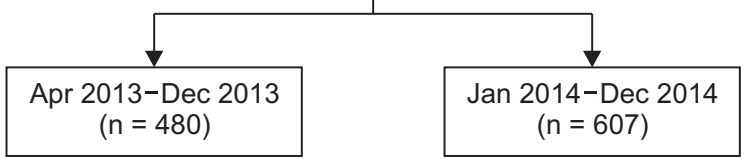

Fig. 1. Patients' flow chart. ICU, intensive care unit.

Table 1. Baseline characteristics of patients of postintervention

\begin{tabular}{lccc}
\hline \multicolumn{1}{c}{ Variable } & April 2013-December 2013 $(\mathrm{n}=480)$ & January 2014-December 2014 $(\mathrm{n}=607)$ & P-value \\
Age $(\mathrm{yr})$ & $60.7 \pm 13.1$ & $61.2 \pm 12.5$ & 0.363 \\
Male sex & $282(58.8)$ & $328(54.0)$ & 0.120 \\
ASA PS classification & $1.8 \pm 0.5$ & $1.7 \pm 0.6$ & 0.217 \\
Types of operation & & & 0.028 \\
Anterior resection & $210(43.8)$ & $213(35.1)$ & $339(55.8)$ \\
Right or left colectomy & $242(50.4)$ & $36(5.9)$ & $7(1.2)$ \\
Segmental resection & $16(3.3)$ & $12(1.8)$ & $109(18.0)$ \\
Subtotal colectomy & $4(0.8)$ & $142 \pm 44$ & 0.268 \\
Total colectomy & $8(1.7)$ & $8.7 \pm 4.7$ & 0.161 \\
Method of operation, open & $75(15.6)$ & & 0.414 \\
Operation time (min) & $147 \pm 47$ & $8.8 \pm 5.1$ &
\end{tabular}

Values are presented as mean \pm standard deviation or number (\%).

ASA, American Society of Anesthesiologist; PS, physical status. 
A

-- - Prophylactic antibiotics within $1 \mathrm{hr}$

- Prophylactic antibiotics selection

-...- Prophylactic antibiotics discontinued within $24 \mathrm{hr}$

...... Appropriate hair removal

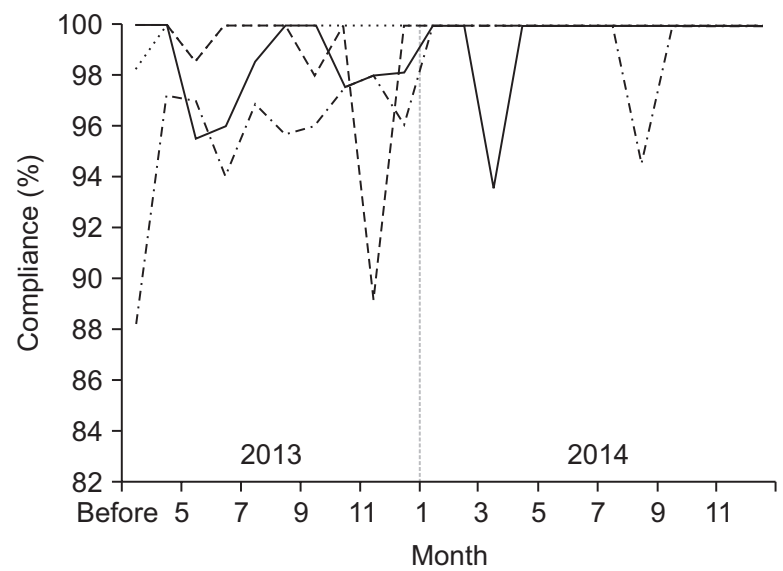

B

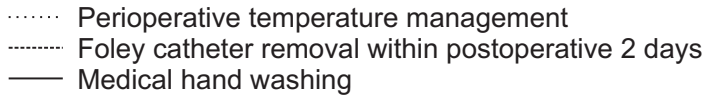

- Medical hand washing

-..- Surgical hand washing

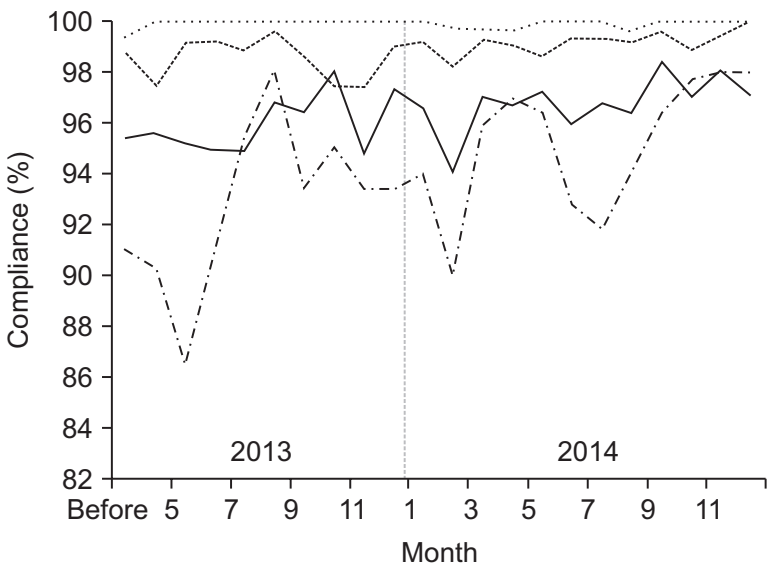

Fig. 2. Compliances of colon surgical site infection bundle (8 elements). (A) Prophylactic antibiotics within 1 hour, prophylactic antibiotics selection, prophylactic antibiotics discontinued within 24 hours, and appropriate hair removal. (B) Perioperative temperature management, Foley catheter removal within postoperative 2 days, medical hand washing, and surgical hand washing.

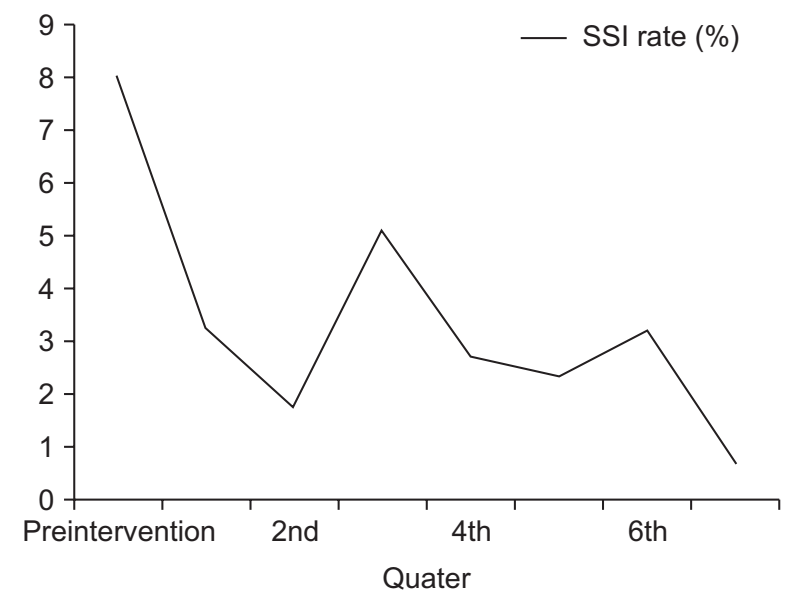

Fig. 3. The quarterly rates of surgical site infections (SSI). Preintervention and postintervention: from 1 st to 7 th quarter.

Table 2. Clinical outcomes of surgical site infections

\begin{tabular}{lcrr}
\hline & \multirow{2}{*}{$\begin{array}{c}\text { Before } \\
\text { Variable }\end{array}$} & \multicolumn{2}{c}{ Year } \\
\cline { 3 - 4 } & $\begin{array}{c}\text { implementation } \\
(\mathrm{n}=150)\end{array}$ & $\begin{array}{c}2013 \\
(\mathrm{n}=480)\end{array}$ & $\begin{array}{c}2014 \\
(\mathrm{n}=607)\end{array}$ \\
\hline Surgical site infection & $12(8.0)$ & $16(3.3)$ & $14(2.3)$ \\
Superficial & $2(1.3)$ & $4(0.8)$ & $3(0.5)$ \\
Deep & $1(0.7)$ & $2(0.4)$ & $1(0.2)$ \\
Organ/space & $9(6.0)$ & $10(2.1)$ & $10(1.6)$ \\
Risk index $\geq 1$ & $7(4.7)$ & $46(9.6)$ & $51(8.4)$ \\
\hline
\end{tabular}

Values are presented as number (\%).



Fig. 4. Comparison of yearly surgical site infections.

(1-year) and 78.9\% (2-year) (Fig. 2B).

A total of 1,237 colorectal surgeries were performed between January 2013 and December 2014 and 42 SSIs (3.9\%) occurred. The rate of SSIs in the preintervention period from January 2013 to March 2013 was 8.0\% $(n=12)$. Fig. 3 shows the rate of SSIs of quarterly reports. Rates of SSIs at postintervention 1-year and 2-year were decreased to 3.3\% $(n=16)$ and 2.3\% $(n=$ 14), respectively, although NNIS risk index for postintervention group was higher than that for the preintervention group (Table 2). In comparison with the rate of SSIs during preintervention, evaluation of outcomes indicated a statistically significant reduction in the rate of SSIs at postintervention 1-year ( $\mathrm{P}=$ 0.019 ) and postintervention 2-year $(\mathrm{P}=0.002)$ (Fig. 4). Patients 
with SSI were associated with significantly longer length of hospital stay compared to patients without SSI (10.47 vs. 8.69 days, $\mathrm{P}=0.049$ ). The most frequently identified pathogens were in the following order; Klebsiella pneumonia, Eggerthella lenta, Escherichia coli, Bacteroid fragilis, and Enterococcus faecium in culture of SSI originated from either the skin or surrounding tissues of the incision, or from deeper structures involved in the operative procedure.

\section{DISCUSSION}

In the present study, we investigated the effect of quality improvement project consisted of multidisciplinary care bundle to reduce SSIs in patients who underwent elective colon surgery. Our results demonstrated that the implementation of the colon SSI bundle was associated with significant decrease of SSI rates despite increase of NNIS risk index. Our study showed that a prolonged length of hospital stay was associated with SSIs.

The potential for the use of care bundles was demonstrated. It reduced complications such as VAP and CRBSI in the critical care population. Because of the high incidence and morbidity of SSIs in the colorectal patient population, colorectal SSIs have been targeted by many hospitals as an area of focus to improve the quality of surgical care. Many hospitals have instituted colorectal SSI bundles in an effort to use evidence-based approaches with a reasonable method to reduce the incidence of SSIs. These bundles, however, have been encountered with varying degrees of success and effectiveness [14-16].

Early studies have found that implementation of the bundle is effective in reducing rates of SSIs. Garcia et al. [17] have described that increasing SCIP compliance in conjunction with a comprehensive unit-based safety program measures consisting of standardized skin preparation, preoperative chlorhexidine showers, selective administration of mechanical bowel preparation, warming of patients in preanesthesia area, and adaptation of enhanced sterile techniques for skin and fascial closure is correlated with decreased SSIs across multiple specialties. Several studies have reported that implementation of bundles of care elements cannot reduce the number of SSIs $[2,4,18,19]$. Tanner et al. [1] described that the Department of Health's High Impact Intervention in England, such as SCIP in the US, was not effective among patients having colorectal surgery. However, only $19 \%$ of patients actually received the entire bundle in their study. Thus, it was impossible to determine the effectiveness of the bundle completely. Hechenbleikner et al. [20] implemented a monitoring tool for measuring compliance of the colorectal bundle and found that most incidences of SSIs at institution occurred in cases with poor compliance to the bundle [2,21]. Having many elements in the care bundle may present inherent defect in implementation and the ability to achieve compliance.
Nevertheless, this may be due to insufficient engagement with staff for the development and implementation of the bundle and inadequate feedback to staff when low compliance rate is identified. Successful implementation of a bundle is dependent on consistent and systematic application of all elements in the bundle. Therefore, compliance with the complete bundle should be reported and SSI rates should be presented for patients who received the entire bundle. In our study, quality improvement team repeatedly explained details of colon SSI bundle for all attending physicians and nurses just before its implementation and after rotation of participants. Except for elements that could be identified by EMR, clinical practice for other elements of bundle, including hair removal using single-use clipper, medical hand washing, and surgical hand washing were directly observed by the assistant. Thus, almost all elements of the bundle were daily monitored except in cases of contact with patients during irregular rounding and wound dressing, which belonged to medical hand washing. The multidisciplinary team met monthly to check the compliance rate of each bundle and to provide feedback results to all attending medical personnel and consequently resulted in change of behavior and attitude. Consequently, we derived high overall compliance of care bundle, although surgeons initially had poor compliance rates to the colon SSI bundle. Improving culture in the operation room, more attention paid by the leadership, improved skill and knowledge of the surgical team, and changed attitude of medical team about hand washing might affect SSI rates which appeared to decrease substantially after the initiation of the colon bundle in our institution.

In a retrospective study using the Premier Perspective Database (Premier Inc., Charlotte, NC, USA) for 405,720 patients from 398 hospitals, the authors documented that there was no relationship between adherence to SCIP process measures and occurrence of SSI [4]. In another study, Pastor et al. [22] showed that compliance with SCIP process measures over 2 consecutive 14-month study periods was significantly increased, although SSIs in patients undergoing colorectal procedure were not meaningfully reduced in their study $(\mathrm{P}<0.92)$. The reason is that some of the SCIP measures might be conducted with a high rate of compliance prior to the introduction of SCIP in 2006. As an example, antibiotics prophylaxis was administered appropriately in all cases in the postintervention period compared with approximately $100 \%$ in the preintervention period in our study. As a result, compliance rate with SCIP measures was increased after implementation. However, the increase was not high enough to observe a significant additional decrease in SSI rate. Consequently, all studies evaluating care bundle should provide compliance data for intervention from both before and after bundle implementation so that the compliance with the bundle can be clearly assessed for effect on SSI. Our study showed compliance of preintervention and 
first and second postintervention, although preintervention period was short. As mentioned above, appropriate prophylactic antibiotics use, appropriate hair removal, and maintenance of normothermia in perioperative period in this study already had high compliance because they had been conducted before implementation of the bundle.

The core elements, including normothermia, glycemic control, timely and antibiotics prophylaxis, and appropriate hair removal, should be considered as they represent baseline characteristics, but these may be insufficient to reduce the overall risk of infection. Therefore, we hypothesized that incorporating multiple strategies with supplemental measures such as medical and surgical hand washings in addition to core elements would have a synergistic effect to reduce SSIs in patients who underwent colon surgery. As much as 50\%-70\% of all healthcare-associated infections are transmitted through the hands of healthcare workers (HCW) [23]. Appropriate hand hygiene of HCWs is the most effective way to prevent and control infections among patients and HCWs [24]. The average compliance of medical personnel to hand washing procedures required before contact with patients was low [25]. Tvedt and Bukholm [26] identified that only 50.4\% of investigated medical personnel performed the recommended hand hygiene procedure while only $20 \%$ performed it correctly. In addition, the frequency of hand hygiene was dependent on factors such as occupation and the nature of the activity. In this present study, medical and surgical hand washings before implementation of the colon SSI bundle were low $74.7 \%$ and $50.0 \%$, respectively). Based on results of direct monitoring by the infection control office in operation room and general ward during study period, we provided feedback to participating colorectal surgeons and nurses. The compliance of medical and surgical hand washings gradually increased every year, although we still did not reach $100 \%$ compliance rate of medical and surgical hand washings. Changing this behavioral aspect requires a change of daily procedures which takes many discussions and repeated feedback [27]. Especially, adherence for each bundle of the bundle would be varied depending on the difficulty in terms of changes of behavior.

To fully appreciate these results, the limitations of this study must be acknowledged. First, we investigated patients who underwent elective colon surgical procedure performed at a single institution. To what extent that similar results could be obtained with the application of the bundle in other population of patients and other institutions is currently unclear. Second, because we focused on the effect of a bundle of care with simultaneous initiation of multiple interventions, it was impossible to evaluate which specific component of the bundle was beneficial. Further study with such bundle to estimate the efficacy of each component is needed to decrease the risk of colorectal SSIs. Third, our study was retrospective study with prospectively collected bundle registry. Because change of behavior is part of the intervention, it is impossible to conduct a randomized controlled study between implementation of bundle and reduction of SSI. Fourth, duration of preintervention was relatively short which might have limited the analysis of process and outcomes. Fifth, elements included in our bundle were slightly different from those used in other studies. However, the bundle approach is believed to be able to create awareness and improve prevention of SSI while medical and surgical hand washing is level 1B evidence for SSI prevention. Sixth, comorbidity and lifestyle, such as obesity, diabetes, cirrhosis, cancer, alcoholism, nutrition, and the immune system, can affect the rates of SSI. However, implementing a colon SSI bundle initially started as a quality improvement project, so we could not investigate detailed information of patients during postintervention, including baseline characteristics of preintervention patients. Follow-up study is needed to evaluate the risk factors of SSI and the applicability of the NNIS risk index for the prediction of SSI. Finally, our study may have possibility of ceiling effect. Prophylactic antibiotics within 1 hour, appropriate hair removal, and maintenance of normothermia in the perioperative period had a high compliance during the entire study period because they had already been conducted at the hospital prior to the implementation of the bundle. However, although the overall process results might be underwhelming and showed a small change in proportion, implementation of colon SSI bundle decreased SSI rate.

In conclusion, after implementation of multidisciplinary care bundle, the compliance of each component was continuously increased and rate of SSI was significantly decreased compared to that before the quality improvement project in patients who underwent elective colon surgery. Behavioral change and improved culture can result in increase of compliance and decrease of SSIs by many discussion and repeated feedback after monitoring. Further study is needed to assess what should be included in the optimal surgical care bundle and determine what level of compliance with bundle measure is needed to achieve good results.

\section{ACKNOWLEDGEMENTS}

\section{Conflict of Interest}

No potential conflict of interest relevant to this article was reported.

\section{ORCID iD}

Chi-Min Park: https://orcid.org/0000-0002-8496-3546

Tae Sun Ha: https://orcid.org/0000-0003-3683-6929

Woo Yong Lee: https://orcid.org/0000-0001-8758-4149

Doo Ryeon Chung: https://orcid.org/0000-0001-9267-101X 
Yoon Ah Park: https://orcid.org/0000-0002-3940-0197

Jong Rim Choi: https://orcid.org/0000-0002-6734-4620

Na Yeon Jeong: https://orcid.org/0000-0001-6939-0391

\section{Author Contribution}

Conceptualization: WYL, DRC

\author{
Formal Analysis: YAP, JRC \\ Investigation: JRC, NYJ \\ Methodology: CMP, TSH \\ Project Administration: YAP, WYL \\ Writing - Original Draft: CMP, TSH \\ Writing - Reivew \& Editing: WYL, DRC, YAP, JRC, NYJ
}

\section{REFERENCES}

1. Tanner J, Padley W, Assadian O, Leaper D, Kiernan M, Edmiston C. Do surgical care bundles reduce the risk of surgical site infections in patients undergoing colorectal surgery? A systematic review and cohort meta-analysis of 8,515 patients. Surgery 2015;158:66-77.

2. Waits SA, Fritze D, Banerjee M, Zhang W, Kubus J, Englesbe MJ, et al. Developing an argument for bundled interventions to reduce surgical site infection in colorectal surgery. Surgery 2014;155:602-6.

3. Arriaga AF, Lancaster RT, Berry WR, Regenbogen SE, Lipsitz SR, Kaafarani HM, et al. The better colectomy project: association of evidence-based best-practice adherence rates to outcomes in colorectal surgery. Ann Surg 2009;250:507-13.

4. Lutfiyya W, Parsons D, Breen J. A colorectal "care bundle" to reduce surgical site infections in colorectal surgeries: a single-center experience. Perm J 2012;16:10-6.

5. Pronovost P, Needham D, Berenholtz S, Sinopoli D, Chu H, Cosgrove S, et al. An intervention to decrease catheter-related bloodstream infections in the ICU. N Engl J Med 2006;355:2725-32.

6. Tanner J, Kiernan M, Hilliam R, Davey S, Collins E, Wood T, et al. Effectiveness of a care bundle to reduce surgical site infections in patients having open colorectal surgery. Ann R Coll Surg Engl 2016;98:270-4.

7. Lee KY, Coleman K, Paech D, Norris S, Tan JT. The epidemiology and cost of surgical site infections in Korea: a systematic review. J Korean Surg Soc 2011;81:295-307. 8. Lee JH, Han HS, Min SK, Lee HK, Lee JH,
Kim YW, et al. Surveillance of surgical wound infections among patients from the department of surgery: prospective trial. Ann Surg Treat Res 2004:66:133-7.

9. Cima R, Dankbar E, Lovely J, Pendlimari $\mathrm{R}$, Aronhalt K, Nehring $\mathrm{S}$, et al. Colorectal surgery surgical site infection reduction program: a national surgical quality improvement program. Driven multidisciplinary single-institution experience. J Am Coll Surg 2013;216:23-33. 10. Wick EC, Hobson DB, Bennett JL, Demski R, Maragakis L, Gearhart SL, et al. Implementation of a surgical comprehensive unit-based safety program to reduce surgical site infections. J Am Coll Surg 2012;215:193-200.

11. Selby LV, Sjoberg DD, Cassella D, Sovel M, Weiser MR, Sepkowitz K, et al. Comparing surgical infections in National Surgical Quality Improvement Project and an Institutional Database. J Surg Res 2015;196:416-20.

12. Gastmeier P, Geffers C, Brandt C, Zuschneid I, Sohr D, Schwab F, et al. Effectiveness of a nationwide nosocomial infection surveillance system for reducing nosocomial infections. J Hosp Infect 2006;64:16-22.

13. Culver DH, Horan TC, Gaynes RP, Martone WJ, Jarvis WR, Emori TG, et al. Surgical wound infection rates by wound class, operative procedure, and patient risk index. National Nosocomial Infections Surveillance System. Am J Med 1991;91:152S-7S.

14. Keenan JE, Speicher PJ, Thacker JK, Walter M, Kuchibhatla M, Mantyh CR. The preventive surgical site infection bundle in colorectal surgery: an effective approach to surgical site infection reduction and health care cost savings. JAMA Surg 2014;149:1045-52.

15. Hawn MT, Vick CC, Richman J, Holman W, Deierhoi RJ, Graham LA, et al. Surgical site infection prevention: time to move beyond the surgical care improvement program. Ann Surg 2011;254:494-9.

16. Berenguer CM, Ochsner MG Jr, Lord SA, Senkowski CK. Improving surgical site infections: using National Surgical Quality Improvement Program data to institute Surgical Care Improvement Project protocols in improving surgical outcomes. J Am Coll Surg 2010;210:737-41, 741-3.

17. Garcia N, Fogel S, Baker C, Remine S, Jones J. Should compliance with the Surgical Care Improvement Project (SCIP) process measures determine Medicare and Medicaid reimbursement rates? Am Surg 2012;78:653-6.

18. Crolla RM, van der Laan L, Veen EJ, Hendriks Y, van Schendel C, Kluytmans J. Reduction of surgical site infections after implementation of a bundle of care. PLoS One 2012;7:e44599.

19. Rasouli MR, Jaberi MM, Hozack WJ, Parvizi J, Rothman RH. Surgical care improvement project (SCIP): has its mission succeeded? J Arthroplasty 2013;28:1072-5.

20. Hechenbleikner EM, Hobson DB, Bennett JL, Wick EC. Implementation of surgical quality improvement: auditing tool for surgical site infection prevention practices. Dis Colon Rectum 2015:58:8390.

21. Bull A, Wilson J, Worth LJ, Stuart RL, 
Gillespie E, Waxman B, et al. A bundle of care to reduce colorectal surgical infections: an Australian experience. J Hosp Infect 2011;78:297-301.

22. Pastor C, Artinyan A, Varma MG, Kim E, Gibbs L, Garcia-Aguilar J. An increase in compliance with the Surgical Care Improvement Project measures does not prevent surgical site infection in colorectal surgery. Dis Colon Rectum 2010;53:24-30.
23. Allegranzi B, Pittet D. Role of hand hygiene in healthcare-associated infection prevention. J Hosp Infect 2009;73:305-15.

24. Pittet D, Hugonnet S, Harbarth S, Mourouga P, Sauvan V, Touveneau S, et al. Effectiveness of a hospital-wide programme to improve compliance with hand hygiene. Infection Control Programme. Lancet 2000;356:1307-12.

25. Garus-Pakowska A, Sobala W, Szatko F. Observance of hand washing procedures performed by the medical personnel before patient contact. Part I. Int J Occup Med Environ Health 2013;26:113-21.

26. Tvedt C, Bukholm G. Alcohol-based hand disinfection: a more robust hand-hygiene method in an intensive care unit. J Hosp Infect 2005:59:229-34.

27. Khan A, Nausheen S. Compliance of surgical hand washing before surgery: role of remote video surveillance. J Pak Med Assoc 2017;67:92-6. 